\section{OPEN ACCESS}

Edited by:

Tharmarajan Ramprasath,

Georgia State University,

United States

Reviewed by:

Nandakumar Natarajan, Department of Surgery, University of

California, San Francisco,

United States

Prabhu Ponnandy,

University of Michigan, United States

Haingo Hantelys,

Georgia State University,

United States

*Correspondence:

Ru Ying

yingru2005@163.com

Specialty section:

This article was submitted to

Cardiovascular Metabolism,

a section of the journal

Frontiers in Cardiovascular Medicine

Received: 01 March 2021

Accepted: 01 April 2021

Published: 28 April 2021

Citation:

Wu C-L, Yin R, Wang S-N and Ying $R$ (2021) A Review of CXCL1 in Cardiac

Fibrosis.

Front. Cardiovasc. Med. 8:674498.

doi: $10.3389 / f c v m .2021 .674498$

\title{
A Review of CXCL1 in Cardiac Fibrosis
}

\author{
Cheng-Long Wu, Ran Yin, Su-Nan Wang and Ru Ying* \\ Department of Cardiology, The First Affiliated Hospital of Nanchang University, Nanchang, China
}

Chemokine C-X-C motif ligand-1 (CXCL1), principally expressed in neutrophils, macrophages and epithelial cells, is a valid pro-inflammatory factor which performs an important role in mediating the infiltration of neutrophils and monocytes/macrophages. Elevated serum level of CXCL1 is considered a pro-inflammatory reaction by the organism. CXCL1 is also related to diverse organs fibrosis according to relevant studies. A growing body of evidence suggests that CXCL1 promotes the process of cardiac remodeling and fibrosis. Here, we review structure and physiological functions of CXCL1 and recent progress on the effects and mechanisms of CXCL1 in cardiac fibrosis. In addition, we explore the role of CXCL1 in the fibrosis of other organs. Besides, we probe the possibility that CXCL1 can be a therapeutic target for the treatment of cardiac fibrosis in cardiovascular diseases.

Keywords: chemokine C-X-C motif ligand-1, cardiac remodeling, cardiac fibrosis, inflammation, atrial fibrillation

\section{HIGHLIGHTS}

- In this review, we have retrospectively analyzed the role of CXCL1 in the pathological process of cardiac fibrosis.

- CXCL1 may be a potential target for the treatment of cardiac fibrosis.

\section{INTRODUCTION}

The morbidity of heart failure (HF) is at a high level. Cardiac remodeling is a clinical process of HF, and could finally evolve into cardiac fibrosis. Cardiac fibrosis usually appears when myocardium is constantly at the stage of ischemia and hypoxia (1). It is proposed that chemokine C-X-C motif ligand-1 (CXCL1) presents significant effect during HF and ischemic cardiomyopathy (2). CXCL1 is a member of chemokines family which mediates the directional immigration of inflammatory cells, and is critical in recruiting neutrophils and monocytes/macrophages into the target position such as injured myocardium and arterial wall in CVD (2). Previous studies show that the inhibition of CXCL1 improves adverse cardiac remodeling and myocardial fibrosis thereby protects cardiac function $(2,3)$. Therefore, novel therapeutic method could be applied based on interference of CXCL1 to improve cardiac fibrosis. CXCL1 is also active in inflammation in other organs as a major neutrophil chemoattractant to mediate tissue injury $(4,5)$. However, the regulation and the mechanism of CXCL1 remain complex and obscure. More research is demanded to clarify CXCL1 and its function in CVD. 


\section{Structure and Physiological Functions of CXCL1}

CXCL1 is widely known as a strong neutrophil chemoattractant which participates in inflammation of multiple tissues $(2,6-$ 8). It is a member of CXC superfamily which is one category of chemokines $(2,9)$. Chemokines are small chemoattractant molecules which recruit and activate leukocytes via specific seven-transmembrane receptors (7). They are classified into four categories according to the sequence of aminoacids related to the first 2 cysteine residues, namely $\mathrm{CC}, \mathrm{CXC}, \mathrm{C}$, and $\mathrm{CX} 3 \mathrm{C}$ families $(7,9,10)$. The CXC family is further divided based on presence or absence of Glu-Leu-Arg sequence (ELR motif) adjacent to CXC motif $(9,11)$. CXCL1 is about $8 \mathrm{kDa}(12$, 13), also called growth-regulated oncogene- $\alpha(\mathrm{GRO}-\alpha)$ and keratinocyte-derived chemokine (KC) (Figure 1) (7, 11), and usually expressed in neutrophils, macrophages, and epithelial cells. CXCL1 produces its effect via its receptor CXCR2 which is mainly expressed on neutrophils and other types of cells (2).

CXCL1 exists in various tissues and produces multiple effects. As a pro-inflammatory chemokine, CXCL1 mediates acute and chronic inflammation in diverse organs thereby promotes the progress of fibrosis. The major physiology function of CXCL1 is mediating leukocyte recruitment and activation to promote inflammation and aggravate tissue injury. ELR-chemokines are valid neutrophil chemoattractants acting on G protein-coupled receptors (11). CXCL1 processes the Glu-Leu-Arg sequence and attracts neutrophils when inflammation occurs (14). Excluding inflammation, CXCL1 is also active in angiogenesis, wound healing as well as tumorogenesis depending on interaction with leukocytes, endothelial cells, and fibroblasts $(7,15)$. CXCL1 is related to angiogenesis during tissue remodeling $(9,16,17)$. Interestingly, ELR-chemokines such as CXCL1 and CXCL2 promote vascular remodeling, whereas non-ELR CXC ligand such as CXCL4 and CXCL9 serve opposite effect (9). Besides, numerous studies indicate CXCL1 is important in tumorogenesis and progress of tumor, and CXCL1 is a promoter in evolvement of some kinds of tumor. In addition, CXCL1 widely participates in wound healing, mitosis and ischemia-reperfusion injury $(18,19)$.

\section{The Role of CXCL1 in the Development of Cardiac Fibrosis}

It is believed that the main pathological characteristics of CVD are cardiac inflammation and fibrosis (20). The pathological characteristics of cardiac fibrosis could be concluded as increased interstitial fibrosis, myocyte death, and cardiac contractile dysfunction (21). Current study indicates that HF could be improved with the depression of cardiac inflammation tends to be closely relevant to the dysfunction of heart. As previously described, CXCL1 is active in mediating the infiltration of neutrophils and monocytes/ macrophages into the impaired tissues including injured myocardium and arteries. It is indicated

Abbreviations: CVD, cardiovascular disease; HF, heart failure; Ang, angiotensin; IL, Interleukin; HSCs, hepatic stellate cells; IHD, ischemic heart disease; RAAS, renin-angiotensin-aldosterone system; MMP-12, matrix metallopeptidase 12; LPS, lipopolysaccharide; CCL, C-C Motif Chemokine Ligand. that CXCL1 may aggravate cardiac fibrosis via pro-inflammatory effect (22). It is found that CXCL1 inhibition protects heart from Ang II-induced inflammation, hypertrophy and fibrosis (2). Mice treated with CXCL1 neutralizing antibody tend to have better cardiac function, lower level of brain natriuretic peptide and less degree of hypertrophy compared with control group. Similarly, cardiac remodeling and fibrosis are apparently alleviated through application of CXCR2 specific inhibitor. In HF patients with hypertension, the serum level of CXCL1 is obviously increased in patient with cardiac remodeling and fibrosis $(2,23)$. It could be concluded that CXCL1 promotes the inflammation in heart thereby accelerates the process of cardiac remodeling and myocardial fibrosis.

Atrial fibrosis is the basis of atrial fibrillation (AF). Current study indicates CXCR-2 knockout mice had significantly attenuated atrial fibrillation inducibility, atrial diameter, fibrosis, and infiltration of macrophages compared with saline-treated wild-type mice (24). Moreover, circulating blood CXCL-1 levels were higher and associated with AF in human patients compared with sinus rhythm controls (24). Besides, CXCL1 is also considered to mediate the irradiated rat cardiac fibrosis by miR21 (25).

In sum, CXCL1 may aggravate cardiac fibrosis induced by hypertension, atrial fibrillation, post-irradiation and so on.

\section{Mechanisms of CXCL1 in Cardiac Fibrosis CXCL1 Mediates Leukocyte Recruitment}

There is growing evidence to support an important role of inflammatory cells especially monocytes/macrophages in the pathophysiology of HF (26). CXCL1 has been reported to exert a critical role in HF by regulating the recruitment of neutrophils, $\mathrm{T}$ lymphocytes and monocytes, especially regulating CXCR2 ${ }^{+}$ monocytes into the heart tissues leading to cardiac remodeling and initiating and developing $\operatorname{AF}(2,24)$. CXCL1 produces its effect via its receptor CXCR2 which is mainly expressed on neutrophils. CXCR2 activation plays critical roles in the recruitment of leucocytes and neutrophils, which are involved in the pathogenesis of atherosclerosis and cardiac fibrosis. In cardiovascular diseases, it is cognized that CXCL1 is vital in enlisting neutrophils and monocytes/macrophages into the impaired myocardium and artery to mediate atherosclerosis and cardiac remodeling (2). Obvious neutrophil influx mediated by CXCL1 is discovered in inflammatory sites (27).

\section{CXCL1 Mediates Inflammation}

The inflammation in plasma and tissue is accompanied with risen level of CXCL1 in relevant studies (28). Some researches demonstrated that the CXCL1-CXCR2 axis mediates the infiltration of monocytes into heart tissues, leading to cardiac fibrosis. CXCL1 is also active in inflammation in other organs as a major neutrophil chemoattractant to mediate tissue injury. CXCL1 may mediates inflammation by NF-кB (nuclear factor$\kappa \mathrm{B})$ signaling, a key regulator of pro-inflammatory mediators and NOX (nicotinamide adenine dinucleotide phosphate oxidase)2 subunit. CXCR-2 deletion reduced the activation of NF$\kappa \mathrm{B}$ signaling (24). However, whether CXCL1 transcription is regulated by $\mathrm{NF}-\kappa \mathrm{B}$ needs further study. 


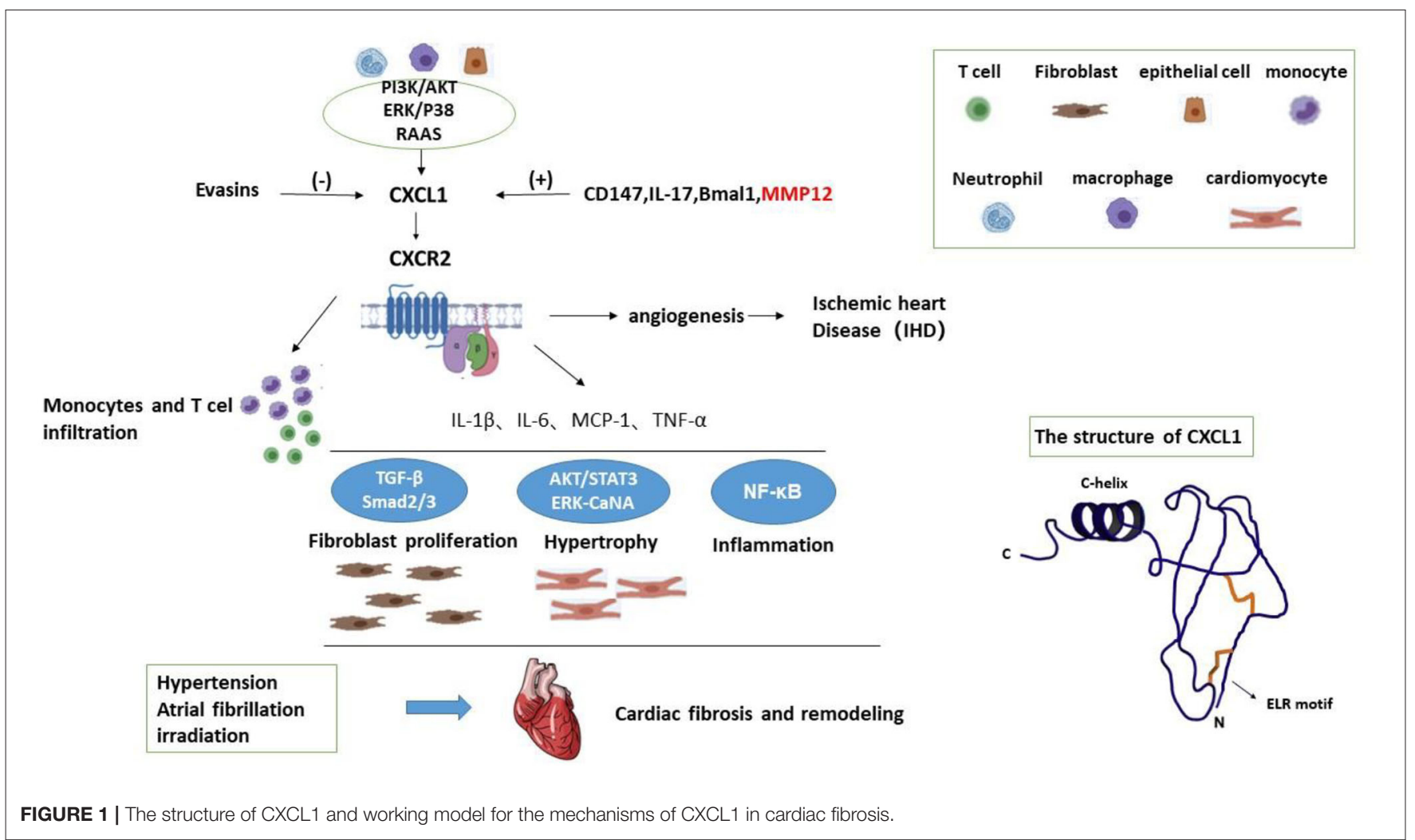

\section{The Effect of CXCR2 on Cardiomyocyte (CM) Hypertrophy}

CXCR2 was reported to promote CM hypertrophy. CXCR2 KO macrophages were co-cultured with WT neonatal rat cardiac myocytes (CMs) or fibroblasts (CFs), respectively. After $24 \mathrm{~h}$ of Ang II treatment, co-culture of CMs with CXCR2 KO macrophages had a significant reduction in CM size, the expression of the protein levels of $\mathrm{p}$-AKT, p-ERK1/2, pSTAT3 and CaNA compared with co-culture of CMs with WT macrophages (2). Therefore, AKT, ERK1/2, STAT3 and CaNA may be the important mediators of cardiomyocyte hypertrophy.

\section{CXCL1 in Cardiovascular Angiogenesis}

Excluding inflammation and fibrosis, CXCL1 is also active in angiogenesis. In chronic ischemic heart disease (IHD) patients, the formation of coronary collateralization is notable. In chronic IHD, myocardial ischemia may stimulate the secretion of angiogenic chemokines such as CXCL1 to mediate the formation of coronary collateralization (9). Coronary collateralization tends to result in better cardiac function, less arrhythmias, less complications and higher survival (9). It is indicated that CXC chemokines play a vital role in the presence of coronary collaterals (9). ELR motif is structurally decisive for the physiological functions of CXC chemokines (9). The level of ELRchemokines including CXCL1 is risen in vascular remodeling and angiogenesis. Conversely, CXC chemokines without ELR motif inhibit the progress of angiogenesis. Therefore, CXCL1 is a potent promoter of angiogenesis.

\section{The Effect of CXCL1 on TGF-Smad2/3 Signaling}

TGF (transforming growth factor)-Smad2/3 are the key signaling mediators of cardiac fibrosis. These researches investigated that TGF- $\beta 1$, p-Smad $2 / 3$ were suppressed in CXCR- 2 knockout mice, and $\alpha$-SMA and collagen I also decreased $(2,24)$. Therefore, CXCL1 may induce cardiac fibrosis by TGF-Smad2/3 signaling.

\section{Several Factors Influence the Level of CXCL1 in Cardiac Fibrosis}

Many factors are known to have an impact on the serum level of CXCL1. Renin-angiotensin-aldosterone system (RAAS) is a major aspect. Angiotensin (Ang) II promotes the expression of CXCL1 (2). The inhibition of the activity of RAAS contributes to cardiac remodeling and fibrosis $(2,3)$. TRAF3IP2 (TRAF3 interacting protein $2 \mathrm{~T}$ ) is known as a signaling intermediate of aldosterone/salt-induced cardiac remodeling and myocardial fibrosis (3). It is discovered that the deletion of TRAF3IP2 gene significantly attenuated expression of CXCL1 (3). Meanwhile, CXCL1 mRNA level increases obviously in aldosterone-treated mice (3). Oppositely, it is indicated that Ang II modulates smoke- induced lung fibrosis and suppresses the increasing of CXCL1 (29).

IL-17 is thought a factor of adverse cardiac remodeling and fibrosis (30), and a key downstream mediator of TGF- $\beta$ which is an important promote-fibrosis cytokine. It is indicated that IL17 promotes the mRNA expression of CXCL1 and CXCL1 is a downstream gene of IL-17 $(30,31)$. With the neutralization of IL-17, there is a downward trend of expression of CXCL1 (30). 
Knockout of IL-17 gene also markedly down-regulates the level of CXCL1 in liver fibrosis (32).

Bmal1 (aryl hydrocarbon receptor nuclear translocator-like protein 1) is the gene of aryl hydrocarbon receptor nuclear translocator-like protein 1 in cardiomyocyte (33). It is presented that cardiomyocyte-specific deletion of Bmall significantly upgrades the transcript level of CXCL1, triggers diastolic dysfunction, extracellular matrix response, and impaired resolution of inflammation (33).

Besides, a proteolytic enzyme named MMP-9 is known to have the ability of decomposing CXCL1 (34). MMP-12 which is produced by macrophages is also related to the serum level of CXCL1 $(35,36)$. It is known for its function of degrading extracellular matrix, but MMP12 has several other functions. The mRNA level of CXCL1 is much higher in MMP12-/- mice in infarct area of heart compared with wild type group (35). It is suggested that MMP12 inhibits infiltration of neutrophils by depression of CXCL1/CXCL2/CXCL5- CXCR2 axis (35). However, in other study, the silence of MMP12 leads to decline of CXCL1 and MMP12 is thought a contributor of secretion of CXCL1 (36). This is a contradiction we should pay attention to.

In addition to the above, a chemokine-binding protein called "Evasins" is demonstrated to have the ability of inhibiting both CC and CXC chemokine (37). Evasin-4 is proved to be relevant to the decreasing of the serum level of CXCL1 (37). CCL5 (C-C Motif Chemokine Ligand 5) is proved important in the synthesis of CXCL1 and it could be blocked by Evasin-4 (37). In addition, chemokines CC-chemokine ligand $2(\mathrm{Ccl} 2)$ was related to the expression of CXCL1 in HSCs (38). In this research, Tnfr $1^{-/-} / \mathrm{Mdr}^{-/-}$mice expressed high levels of CCL2, showed significantly up-regulated hepatic gene expression of CXCL1 compared to wild type.

\section{Signaling Pathway Regulating CXCL1 Expression}

In addition to neutrophils, macrophages, and epithelial cells, activated hepatic stellate cells (HSCs) also release CXCL1. CD147 promotes CXCL1 expression in HSCs and CXCL1 promoted HSCs activation through autocrine (39). CD147 can bind to integrin and activates the downstream FAK/PI3K signaling pathway. CD147 overexpression induced the AKT phosphorylation; however, treating with FAK/PI3K inhibitor LY294002, CD147-induced AKT phosphorylation and CXCL1 expression were significantly inhibited. Taken together, CD147 regulates CXCL1 release in HSCs by phosphatidylinositol 3 kinase $(\mathrm{PI} 3 \mathrm{~K}) /$ protein kinase $\mathrm{B}(\mathrm{AKT})$ signaling. Therefore, PI3K/AKT signaling may be one mechanism of CXCL1 expression. A working model is illustrated in Figure 1.

MMP12 (matrix metallopeptidase 12) is proved relevant to several inflammatory diseases. Increased expression of MMP12 leads to proliferation of macrophages (36). CXCL1 is regulated by MMP12 as a pro-inflammatory chemokine (36). Knockdown of MMP12 gene reduces the expression of multiple types of cytokines including CXCL1 in animal models (36). Notably, MMP12 silencing significantly down-regulate the expression of mitogen-activated protein kinase p38(P38) and extracellular regulated kinase $1 / 2(\mathrm{ERK} 1 / 2)$ and their phosphorylation (36). It is hard to say the role of the ERK/P38 MAPK signaling pathway in pro-inflammatory chemokine CXCL1 during inflammation, but it provides a new mentality to figure out CXCL1 and its regulation.

\section{CXCL1 in the Fibrosis of Other ORGANS Lung Fibrosis}

CXCL1 is connected with fibrosis of other organs in extensive research. Lung fibrosis is known as a progressive disease characterized by inflammatory infiltration and interstitial fibrosis (40, 41). Neutrophils mediate lung injury via recruitment and activation $(42,43)$. Neutrophil influx correlated with CXCL1 plays an important role in pulmonary fibrosis (27, 28). Inhibition of the genetic synthesis of extracellular matrix protein is accompanied with reduced level of CXCL1 $(44,45)$. It is suggested that IL-17 inducing chemokines promote the progress of pulmonary fibrosis (46). CXCL1 is recognized as the downstream gene regulated by IL-17 and is at a higher level in fibrosis model (46-48). IL-9 is similar to IL-17 in some experiments $(49,50)$. In bleomycin-induced lung fibrosis model, the release of CXCL1 is promoted by bleomycin $(51,52)$. Besides, CXCL1 is a novel marker for evaluating the severity of chronic obstructive pulmonary disease $(28,53)$. CXCL1 is also involved in airway remodeling, asthma and cystic fibrosis in lung (54-56).

\section{Hepatic Fibrosis}

CXCL1 is also related to hepatic fibrosis. Similar to pulmonary fibrosis, liver fibrosis also has an accumulation of extracellular matrix proteins in structure (57-59). Fibrosis in liver is usually a consequence of chronic inflammation caused by alcohol, diet and virus infections $(39,60)$. Neutrophils play an important role in liver injury (61). Cholestatic liver injury is accompanied with infiltration of neutrophils and upregulated expression of CXCL1 $(62,63)$. Decreased level of CXCL1 is observed in treatment of non-alcoholic steatohepatitis and hepatic fibrosis. Hepatic stellate cells (HSCs) are considered as a precursor of myofibroblasts in the liver and correlate with the production of extracellular matrix (64-66). CXCL1 could be secreted by HSCs in liver and CXCL1 promotes the activation of HSCs at the same time (39). HSCs stimulated by CXCL1 increase the expression of collagen type I and $\alpha$-SMA (39). Therefore, CXCL1 is considered to be a promoter of hepatic fibrosis (67). Reduced expression CXCL1 is able to accelerate HSCs apoptosis thereby ameliorating liver fibrosis (68). In addition, CXCL1 performs a vital role in hepatic fibrosis induced by high cholesterol and alcohol. Blockade of CXCL1 by specific antibody reduced hepatic neutrophil infiltration and liver fibrosis in relevant study (66). Besides, M2-type macrophage mediated by CXCL1 is significantly upregulated in cirrhosis patients (69). CXCL1 could also be regulated by IL-17 in liver (70). Extracellular vesicles-derived miR-150-5p secreted by adiposederived mesenchymal stem cells inhibits CXCL1 expression to attenuate hepatic fibrosis (5). Surprisingly, some studies suggest that CXCL1 has a protective effect against liver fibrosis and the expression of CXCL1 suppress fibrosis (58). The effect of CXCL1 in hepatic fibrosis remains further research. 


\section{Renal Fibrosis}

All progressive chronic kidney diseases including obstructive nephropathy could result to renal fibrosis with impaired renal function $(71,72)$. CXCL1 acts an important role in chronic renal inflammation including obstruction as a neutrophil chemoattractant (73-75). Increased expression of CXCL1 exacerbates kidney damage in animal model $(4,76)$. The expression of CXCL1 gene could be controlled by NF- $\kappa \mathrm{B}$ in kidney, and NF-кB is known as an indirect pro-fibrogenic factor (77). Blockade of CXCL1-CXCR2 axis efficiently alleviated renal inflammation. In addition, previous studies indicated that renal fibrosis is prompted by increased level of receptor CXCR2 (78). Macrophages mediated by CXCL1 are correlated with collagen formation, extracellular matrix deposition and the degree of renal dysfunction in experimental animal models (79). CXCL1 is also relevant to the fibrosis of intra-allograft in some cases (80).

\section{Other Organs}

CXCL1 is also related to the fibrosis in pancreas and biliary atresia $(81,82)$. What's more, fibrosis after autoimmune thyroiditis is also supposed relevant to CXCL1 (83).

\section{CONCLUSION}

CXCL1 is a potent neutrophil chemoattractant, which could be secreted by macrophages, fibroblasts, keratinocytes, and epithelial cells (84). It is involved in diverse tissue inflammations, fibrosis, tumor, angiogenesis in various tissues. In CVD, CXCL1 plays an important role in cardiac fibrosis especially induced by hypertension, atrial fibrillation, post-irradiation. Therefore, CXCL1 is a promising target and antagonism of CXCL1 or CXCR2 is a novel therapy for treatment of cardiac fibrosis. However, whether cardiac fibrosis induced by other causes (such

\section{REFERENCES}

1. Lafuse WP, Wozniak DJ, Rajaram M. Role of cardiac macrophages on cardiac inflammation, fibrosis and tissue repair. Cells. (2020) 10:51. doi: 10.3390/cells10010051

2. Wang L, Zhang YL, Lin QY, Liu Y, Guan XM, Ma XL, et al. CXCL1-CXCR2 axis mediates angiotensin II-induced cardiac hypertrophy and remodelling through regulation of monocyte infiltration. Eur Heart J. (2018) 39:181831. doi: 10.1093/eurheartj/ehy085

3. Sakamuri SS, Valente AJ, Siddesha JM, Delafontaine P, Siebenlist U, Gardner JD, et al. TRAF3IP2 mediates aldosterone/salt-induced cardiac hypertrophy and fibrosis. Mol Cell Endocrinol. (2016) 429:84-92. doi: 10.1016/j.mce.2016.03.038

4. Hermert D, Martin IV, Reiss LK, Liu X, Breitkopf DM, Reimer KC, et al. The nucleic acid binding protein YB-1-controlled expression of CXCL1 modulates kidney damage in liver fibrosis. Kidney Int. (2020) 97:74152. doi: 10.1016/j.kint.2019.10.024

5. Du Z, Wu T, Liu L, Luo B, Wei C. Extracellular vesicles-derived miR150-5p secreted by adipose-derived mesenchymal stem cells inhibits CXCL1 expression to attenuate hepatic fibrosis. J Cell Mol Med. (2021) 25:70115. doi: 10.1111/jcmm.16119

6. Cabrera S, Maciel M, Herrera I, Nava T, Vergara F, Gaxiola M, et al. Essential role for the ATG4B protease and autophagy in bleomycin-induced pulmonary fibrosis. Autophagy. (2015) 11:670-84. doi: 10.1080/15548627.2015.1034409 as lone AF, aortic coarctation and myocardial infarction) is associated with CXCL1 is not clear. Hence, more researches need to conduct to ascertain the roles and relevant mechanisms of CXCL1 in these cardiac fibrosis. Actually, we have been doing related research, and we found that the oxidative stress related pathway might contribute to the effect of CXCL1 in cardiac fibrosis. However, we need more research. With the aid of further researches of CXCL1, it will help cardiologists devise more reasonable therapeutic plans and improve the prognosis of CVD patients.

\section{DATA AVAILABILITY STATEMENT}

The raw data supporting the conclusions of this article will be made available by the authors, without undue reservation.

\section{AUTHOR CONTRIBUTIONS}

C-LW: the main writer. RYin: reference collection and screening. S-NW: typography and format modification. RYing: manuscript design, modification, and submission. All authors contributed to the article and approved the submitted version.

\section{FUNDING}

This research work was financially supported by grants: the National Natural Science Foundation of China (81960093), Science and Technology Program of Jiangxi Province (No. 20171BAB215003), Science and Technology Plan of Health Commission of Jiangxi Province (202130208), and Science and Technology Project of Education Department of Jiangxi Province (GJJ180044).
7. Russo RC, Guabiraba R, Garcia CC, Barcelos LS, Roffe E, Souza AL, et al. Role of the chemokine receptor CXCR2 in bleomycin-induced pulmonary inflammation and fibrosis. Am J Respir Cell Mol Biol. (2009) 40:41021. doi: 10.1165/rcmb.2007-0364OC

8. Jimenez Calvente $C$, Del Pilar $H$, Tameda $M$, Johnson CD, Feldstein AE. MicroRNA $2233 \mathrm{p}$ negatively regulates the NLRP3 inflammasome in acute and chronic liver injury. Mol Ther. (2020) 28:653-63. doi: 10.1016/j.ymthe.2019.09.013

9. Keeley EC, Moorman JR, Liu L, Gimple LW, Lipson LC, Ragosta $\mathrm{M}$, et al. Plasma chemokine levels are associated with the presence and extent of angiographic coronary collaterals in chronic ischemic heart disease. PLoS ONE. (2011) 6:e21174. doi: 10.1371/journal.pone. 0021174

10. Zhu R, Nasu K, Aoyagi Y, Hirakawa T, Takebayashi K, Narahara H. Chemokine expression profiles of ovarian endometriotic stromal cells in three-dimensional culture. J Reprod Immunol. (2020) 138:103100. doi: 10.1016/j.jri.2020.103100

11. Besnard AG, Struyf S, Guabiraba R, Fauconnier L, Rouxel N, Proost $\mathrm{P}$, et al. CXCL6 antibody neutralization prevents lung inflammation and fibrosis in mice in the bleomycin model. J Leukoc Biol. (2013) 94:131723. doi: $10.1189 / \mathrm{jlb} .0313140$

12. Hong SN, Joung J, Bae JS, Lee CS, Koo JS, Park SJ, et al., and Kim Y, RNA-seq reveals transcriptomic differences in inflamed and noninflamed intestinal mucosa of Crohn's disease patients compared with 
normal mucosa of healthy controls. Inflamm Bowel Dis. (2017) 23:1098108. doi: 10.1097/MIB.0000000000001066

13. Ye MY, Chen MY, Chang YH, Huang JS, Huang TT, Wong TY, et al. Growthregulated oncogene- $\alpha$ from oral submucous fibrosis fibroblasts promotes malignant transformation of oral precancerous cells. J Oral Pathol Med. (2018) 47:880-6. doi: 10.1111/jop.12768

14. Konishi T, Schuster RM, Goetzman HS, Caldwell CC, Lentsch AB. Cellspecific regulatory effects of CXCR2 on cholestatic liver injury. Am J Physiol Gastrointest Liver Physiol. (2019) 317:G773-83. doi: 10.1152/ajpgi.000 80.2019

15. Wilson CL, Jurk D, Fullard N, Banks P, Page A, Luli S, et al. NFkappaB1 is a suppressor of neutrophil-driven hepatocellular carcinoma. Nat Commun. (2015) 6:6818. doi: 10.1038/ncomms7818

16. Tsai $\mathrm{Y}, \mathrm{Hao} \mathrm{S}$, Chen $\mathrm{C}$, Lin BJ, Wu W. Involvement of B2 receptor in bradykinin-induced proliferation and proinflammatory effects in human nasal mucosa-derived fibroblasts isolated from chronic rhinosinusitis patients. PLOS ONE. (2015) 10:e0126853. doi: 10.1371/journal.pone. 0126853

17. Kang J, Hur J, Kang JA, Lee HS, Jung H, Choi JI, et al. Priming mobilized peripheral blood mononuclear cells with the "activated platelet supernatant" enhances the efficacy of cell therapy for myocardial infarction of rats. Cardiovasc Ther. (2016) 34:245-53. doi: 10.1111/1755-59 22.12194

18. Amrouche L, Desbuissons G, Rabant M, Sauvaget V, Nguyen C, Benon A, et al. MicroRNA-146a in human and experimental ischemic AKI: CXCL8-dependent mechanism of action. J Am Soc Nephrol. (2017) 28:47993. doi: 10.1681/ASN.2016010045

19. Perrault DP, Lee GK, Bouz A, Sung C, Yu R, Pourmoussa AJ, et al. Ischemia and reperfusion injury in superficial inferior epigastric artery-based vascularized lymph node flaps. PLOS ONE. (2020) 15:e0227599. doi: 10.1371/journal.pone.0227599

20. Jin $\mathrm{Y}, \mathrm{Wu} \mathrm{Z}$, Wang $\mathrm{N}$, Duan $\mathrm{S}$, Wu Y, Wang J, et al. Association of EGF receptor and NLRs signaling with cardiac inflammation and fibrosis in mice exposed to fine particulate matter. J Biochem Mol Toxicol. (2016) 30:42937. doi: $10.1002 /$ jbt.21806

21. Zhang Y, Geng C, Yang J, Fang J, Yan X, Li P, et al. Chronic inhibition of chemokine receptor CXCR2 attenuates cardiac remodeling and dysfunction in spontaneously hypertensive rats. Biochim Biophys Acta. (2019) 1865:165551. doi: 10.1016/j.bbadis.2019.165551

22. Ferreira JP, Pizard A, Machu J, Bresso E, Rocca HB, Girerd N, et al. Plasma protein biomarkers and their association with mutually exclusive cardiovascular phenotypes: the FIBRO-TARGETS case-control analyses. Clin Res Cardiol. (2020) 109:22-33. doi: 10.1007/s00392-01901480-4

23. Mikolajczyk TP, Szczepaniak P, Vidler F, Maffia P, Graham GJ, Guzik TJ. Role of inflammatory chemokines in hypertension. Pharmacol Therap. (2021) 223:107799. doi: 10.1016/j.pharmthera.2020.107799

24. Zhang Y, Cao H, Han X, Teng F, Chen C, Yang J, et al. Chemokine receptor CXCR-2 initiates atrial fibrillation by triggering monocyte mobilization in mice. Hypertension. (2020) 76:381-92. doi: 10.1161/HYPERTENSIONAHA.120.14698

25. Guo H, Zhao X, Su H, Ma C, Liu K, Kong S, et al. miR-21 is upregulated, promoting fibrosis and blocking $\mathrm{G} 2 / \mathrm{M}$ in irradiated rat cardiac fibroblasts. PeerJ. (2020) 8:e10502. doi: 10.7717/peerj.10502

26. Wrigley BJ, Lip GY, Shantsila E. The role of monocytes and inflammation in the pathophysiology of heart failure. Eur J Heart Fail. (2011) 13:116171. doi: 10.1093/eurjhf/hfr122

27. Leal MP, Brochetti RA, Ignácio A, Câmara NOS, Da Palma RK, de Oliveira LVF, et al. Effects of formaldehyde exposure on the development of pulmonary fibrosis induced by bleomycin in mice. Toxicol Rep. (2018) 5:512-20. doi: 10.1016/j.toxrep.2018.03.016

28. Lee SY, Cho JH, Cho SS, Bae CS, Kim GY, Park DH. Establishment of a chronic obstructive pulmonary disease mouse model based on the elapsed time after LPS intranasal instillation. Lab Anim Res. (2018) 34:110. doi: 10.5625/lar.2018.34.1.1

29. Zhang Y, Li Y, Shi C, Fu X, Zhao L, Song Y. Angiotensin-(1-7)-mediated Mas1 receptor/NF-кB-p65 signaling is involved in a cigarette smoke-induced chronic obstructive pulmonary disease mouse model. Environ Toxicol. (2018) 33:5-15. doi: 10.1002/tox.22454

30. Chang SL, Hsiao YW, Tsai YN, Lin SF, Liu SH, Lin YJ, et al. Interleukin-17 enhances cardiac ventricular remodeling via activating MAPK pathway in ischemic heart failure. J Mol Cell Cardiol. (2018) 122:6979. doi: 10.1016/j.yjmcc.2018.08.005

31. Liu CL, Shi GP. Calcium-activated chloride channel regulator 1 (CLCA1): more than a regulator of chloride transport and mucus production. World Allergy Organ J. (2019) 12:100077. doi: 10.1016/j.waojou.2019.100077

32. Zhang Y, Huang D, Gao W, Yan J, Zhou W, Hou X, et al. Lack of IL-17 signaling decreases liver fibrosis in murine schistosomiasis japonica. Int Immunol. (2015) 27:317-25. doi: 10.1093/intimm/ dxv017

33. Ingle KA, Kain $\mathrm{V}$, Goel $\mathrm{M}$, Prabhu SD, Young ME, Halade GV. Cardiomyocyte-specific Bmall deletion in mice triggers diastolic dysfunction, extracellular matrix response, and impaired resolution of inflammation. Am J Physiol Heart Circulatory Physiol. (2015) 309:H1827-36. doi: 10.1152/ajpheart.00608.2015

34. Ilatovskaya DV, Pitts C, Clayton J, Domondon M, Troncoso M, Pippin S, et al. $\mathrm{CD} 8(+)$ T-cells negatively regulate inflammation post-myocardial infarction. Am J Physiol Heart Circ Physiol. (2019) 317:H581-96. doi: 10.1152/ajpheart.00112.2019

35. Kubota A, Suto A, Suzuki K, Kobayashi Y, Nakajima H. Matrix metalloproteinase-12 produced by Ly6Clow macrophages prolongs the survival after myocardial infarction by preventing neutrophil influx. J Mol Cell Cardiol. (2019) 131:41-52. doi: 10.1016/j.yjmcc.2019. 04.007

36. Guan C, Xiao Y, Li K, Wang T, Liang Y, Liao G. MMP-12 regulates proliferation of mouse macrophages via the ERK/P38 MAPK pathways during inflammation. Exp Cell Res. (2019) 378:182-90. doi: 10.1016/j.yexcr.2019.03.018

37. Braunersreuther V, Montecucco F, Pelli G, Galan K, Proudfoot AE, Belin A, et al. Treatment with the CC chemokine-binding protein Evasin-4 improves post-infarction myocardial injury and survival in mice. Thromb Haemost. (2013) 110:807 doi: 10.1160/TH13-04-0297

38. Berkhout L, Barikbin R, Schiller B, Ravichandran G, Krech T, Neumann K, et al. Deletion of tumour necrosis factor $\alpha$ receptor 1 elicits an increased TH17 immune response in the chronically inflamed liver. Sci Rep. (2019) 9:4232. doi: 10.1038/s41598-019-40324-z

39. Shi W, Ju D, Li H, Yuan L, Cui J, Luo D, et al. CD147 promotes CXCL1 expression and modulates liver fibrogenesis. Int J Mol Sci. (2018) 19:1145. doi: 10.3390/ijms19041145

40. Brochetti RA, Leal MP, Rodrigues R, Da Palma RK, de Oliveira LVF, Horliana ACRT, et al. Photobiomodulation therapy improves both inflammatory and fibrotic parameters in experimental model of lung fibrosis in mice. Lasers Med Sci. (2017) 32:1825-34. doi: 10.1007/s10103-017-2281-z

41. Nakazawa Y, Ohtsuka S, Nakahashi-Oda C, Shibuya A. Cutting edge: involvement of the immunoreceptor $\mathrm{CD} 300 \mathrm{c} 2$ on alveolar macrophages in bleomycin-induced lung fibrosis. J Immunol. (2019) 203:3107-11. doi: 10.4049/jimmunol.1900890

42. Mo Y, Jiang M, Zhang Y, Wan R, Li J, Zhong CJ, et al. Comparative mouse lung injury by nickel nanoparticles with differential surface modification. $J$ Nanobiotechnology. (2019) 17:2. doi: 10.1186/s12951-018-0436-0

43. Wan R, Mo Y, Zhang Z, Jiang M, Tang S, Zhang Q. Cobalt nanoparticles induce lung injury, DNA damage and mutations in mice. Part Fibre Toxicol. (2017) 14:38. doi: 10.1186/s12989-017-0219-z

44. Liu G, Cooley MA, Jarnicki AG, Hsu AC, Nair PM, Haw TJ, et al. Fibulin1 regulates the pathogenesis of tissue remodeling in respiratory diseases. JCI Insight. (2016) 1:e86380. doi: 10.1172/jci.insight.86380

45. Liu G, Cooley MA, Nair PM, Donovan C, Hsu AC, Jarnicki AG, et al. Airway remodelling and inflammation in asthma are dependent on the extracellular matrix protein fibulin-1c. J Pathol. (2017) 243:510-23. doi: 10.1002/pa th. 4979

46. Cipolla E, Fisher AJ, Gu H, Mickler EA, Agarwal M, Wilke CA, et al. IL-17A deficiency mitigates bleomycin-induced complement activation during lung fibrosis. FASEB J. (2017) 31:5543-56. doi: 10.1096/fj.2017 00289R 
47. Martinu T, McManigle WC, Kelly FL, Nelson ME, Sun J, Zhang HL, et al. IL-17A contributes to lung fibrosis in a model of chronic pulmonary graft-versus-host disease. Transplantation. (2019) 103:226474. doi: 10.1097/TP.0000000000002837

48. Slivka PF, Hsieh CL, Lipovsky A, Pratt SD, Locklear J, Namovic MT, et al. Small molecule and pooled CRISPR screens investigating IL17 signaling identify BRD2 as a novel contributor to keratinocyte inflammatory responses. ACS Chem Biol. (2019) 14:857-72. doi: 10.1021/acschembio. 8 b00260

49. Sugimoto N, Suzukawa M, Nagase H, Koizumi Y, Ro S, Kobayashi K, et al. IL-9 blockade suppresses silica-induced lung inflammation and fibrosis in mice. Am J Respir Cell Mol Biol. (2019) 60:232-43. doi: 10.1165/rcmb.20170287OC

50. Esnault S, Bernau K, Torr EE, Bochkov YA, Jarjour NN, Sandbo N. RNA-sequencing analysis of lung primary fibroblast response to eosinophil-degranulation products predicts downstream effects on inflammation, tissue remodeling and lipid metabolism. Respir Res. (2017) 18:188. doi: 10.1186/s12931-017-0669-8

51. Pereira PR, Oliveira-Junior MC, Mackenzie B, Chiovatto JE, Matos Y, Greiffo FR, et al. Exercise reduces lung fibrosis involving serotonin/Akt signaling. Med Sci Sports Exerc. (2016) 48:1276-84. doi: 10.1249/MSS.0000000000000907

52. Andrade-Sousa AS, Rogerio PP, MacKenzie B, Oliveira-Junior MC, Assumpcao-Neto E, Brandao-Rangel MA, et al. Aerobic exercise attenuated bleomycin-induced lung fibrosis in Th2-dominant mice. PLoS ONE. (2016) 11:e0163420. doi: 10.1371/journal.pone.0163420

53. Lee SJ, Park JH, Lee JY, Jeong YJ, Song JA, Lee K, Kim DJ. Establishment of a mouse model for pulmonary inflammation and fibrosis by intratracheal instillation of polyhexamethyleneguanidine phosphate. J Toxicol Pathol. (2016) 29:95-102. doi: 10.1293/tox.2015-0067

54. Dennis EA, Coats MT, Griffin SE, Hale JY, Novak L, Briles DE, Crain MJ. The effects of CFTR and mucoid phenotype on susceptibility and innate immune responses in a mouse model of pneumococcal lung disease. PLoS ONE. (2015) 10:e0140335. doi: 10.1371/journal.pone.0140335

55. Stephenson ST, Brown LAS, Helms MN, Qu H, Brown SD, Brown MR, et al. Cysteine oxidation impairs systemic glucocorticoid responsiveness in children with difficult-to-treat asthma. J Allergy Clin Immunol. (2015) 136:454-61.e9. doi: 10.1016/j.jaci.2015.01.023

56. Bartling TR, Drumm ML. Oxidative stress causes IL8 promoter hyperacetylation in cystic fibrosis airway cell models. Am J Respir Cell Mol Biol. (2009) 40:58-65. doi: 10.1165/rcmb.2007-0464OC

57. Cannito S, Milani C, Cappon A, Parola M, Strazzabosco M, Cadamuro M. Fibroinflammatory liver injuries as preneoplastic condition in cholangiopathies. Int J Mol Sci. (2018) 19:3875. doi: 10.3390/ijms19123875

58. Johansson S, Talloen W, Tuefferd M, Darling JM, Scholliers A, Fanning G, et al. Plasma levels of growth-related oncogene (CXCL1-3) associated with fibrosis and platelet counts in HCV-infected patients. Aliment Pharmacol Ther. (2015) 42:1111-21. doi: 10.1111/apt.13389

59. He L, Ye X, Gao M, Yang J, Ma J, Xiao F, et al. Down-regulation of GLT25D1 inhibited collagen secretion and involved in liver fibrogenesis. Gene. (2020) 729:144233. doi: 10.1016/j.gene.2019.144233

60. Robert S, Gicquel T, Bodin A, Lagente V, Boichot E. Characterization of the MMP/TIMP imbalance and collagen production induced by IL-1 $\beta$ or TNF- $\alpha$ release from human hepatic stellate cells. PLoS ONE. (2016) 11:e0153118. doi: 10.1371/journal.pone.0153118

61. Chen L, Gu J, Qian Y, Li M, Qian Y, Xu M, et al. Deletion of C-C motif chemokine ligand 5 worsens invariant natural killer T-cell-mediated hepatitis via compensatory up-regulation of CXCR2-related chemokine activity. Cell Mol Gastroenterol Hepatol. (2019) 7:623-39. doi: 10.1016/j.jcmgh.2018.12.009

62. Gehrke N, Nagel M, Straub BK, Worns MA, Schuchmann M, Galle PR, et al. Loss of cellular FLICE-inhibitory protein promotes acute cholestatic liver injury and inflammation from bile duct ligation. Am J Physiol Gastrointest Liver Physiol. (2018) 314:G319-33. doi: 10.1152/ajpgi.00097.2017

63. Morrison MC, Liang W, Mulder P, Verschuren L, Pieterman E, Toet K, et al. Mirtoselect, an anthocyanin-rich bilberry extract, attenuates non-alcoholic steatohepatitis and associated fibrosis in ApoE(*)3Leiden mice. J Hepatol. (2015) 62:1180-6. doi: 10.1016/j.jhep.2014.12.011

64. Pulli B, Ali M, Iwamoto Y, Zeller MWG, Schob S, Linnoila JJ, Chen JW. Myeloperoxidase-hepatocyte-stellate cell cross talk promotes hepatocyte injury and fibrosis in experimental nonalcoholic steatohepatitis. Antioxid Redox Signal. (2015) 23:1255-69. doi: 10.1089/ars.2014.6108

65. Yang YM, Fukui M, Wang Z, Miao F, Karriker MJ, Seki E. Interventional potential of recombinant feline hepatocyte growth factor in a mouse model of non-alcoholic steatohepatitis. Front Endocrinol. (2018) 9:378. doi: 10.3389/fendo.2018.00378

66. Zhou Z, Xu MJ, Cai Y, Wang W, Jiang JX, Varga ZV, et al. Neutrophil-hepatic stellate cell interactions promote fibrosis in experimental steatohepatitis. Cell Mol Gastroenterol Hepatol. (2018) 5:399-413. doi: 10.1016/j.jcmgh.2018.01.003

67. Yang L, Miura K, Zhang B, Matsushita H, Yang YM, Liang $\mathrm{S}$, et al. TRIF differentially regulates hepatic steatosis and inflammation/fibrosis in mice. Cell Mol Gastroenterol Hepatol. (2017) 3:469-83. doi: 10.1016/j.jcmgh.2016.12.004

68. Zubiete-Franco I, Fernandez-Tussy P, Barbier-Torres L, Simon J, FernandezRamos D, Lopitz-Otsoa F, et al. Deregulated neddylation in liver fibrosis. Hepatology. (2017) 65:694-709. doi: 10.1002/hep.28933

69. Karababa A, Groos-Sahr K, Albrecht U, Keitel V, Shafigullina A, Gorg B, Haussinger D. Ammonia attenuates LPS-induced upregulation of pro-inflammatory cytokine mRNA in co-cultured astrocytes and microglia. Neurochem Res. (2017) 42:737-49. doi: 10.1007/s11064-0162060-4

70. Ma HY, Yamamoto G, Xu J, Liu X, Karin D, Kim JY, et al. IL-17 signaling in steatotic hepatocytes and macrophages promotes hepatocellular carcinoma in alcohol-related liver disease. J Hepatol. (2019) 72:94659. doi: 10.1016/j.jhep.2019.12.016

71. Qiu S, Xiao Z, Piao C, Zhang J, Dong Y, Cui W, et al. AMPKalpha2 reduces renal epithelial transdifferentiation and inflammation after injury through interaction with CK2beta. J Pathol. (2015) 237:330-42. doi: 10.1002/ path. 4579

72. Stribos E, Seelen MA, van Goor H, Olinga P, Mutsaers H. Murine Precision-cut kidney slices as an ex vivo model to evaluate the role of transforming growth factor-betal signaling in the onset of renal fibrosis. Front Physiol. (2017) 8:1026. doi: 10.3389/fphys.201 7.01026

73. Ghosh M, Thangada S, Dasgupta O, Khanna KM, Yamase HT, Kashgarian M, et al. Cell-intrinsic sphingosine kinase 2 promotes macrophage polarization and renal inflammation in response to unilateral ureteral obstruction. PLoS ONE. (2018) 13:e0194053. doi: 10.1371/journal.pone.0194053

74. Zhao Y, Zhu L, Zhou T, Zhang Q, Shi S, Liu L, et al. Urinary CXCL1: a novel predictor of IgA nephropathy progression. PLoS ONE. (2015) 10:e0119033. doi: 10.1371/journal.pone.0119033

75. Ye Y, Zhang Y, Wang B, Walana W, Wei J, Gordon JR, et al. CXCR1/CXCR2 antagonist G31P inhibits nephritis in a mouse model of uric acid nephropathy. Biomed Pharmacother. (2018) 107:1142-50. doi: 10.1016/j.biopha.2018.07.077

76. Ravichandran K, Wang Q, Ozkok A, Jani A, Li H, He Z, et al. CD4 T cell knockout does not protect against kidney injury and worsens cancer. J Mol Med. (2016) 94:443-55. doi: 10.1007/s00109-015-1366-Z

77. Wang DT, Huang RH, Cheng X, Zhang ZH, Yang YJ, Lin X. Tanshinone IIA attenuates renal fibrosis and inflammation via altering expression of TGFbeta/Smad and NF-kappaB signaling pathway in 5/6 nephrectomized rats. Int Immunopharmacol. (2015) 26:4-12. doi: 10.1016/j.intimp.2015.02.027

78. Wang D, Chen X, Fu M, Xu H, Li Z. Tacrolimus increases the expression level of the chemokine receptor CXCR2 to promote renal fibrosis progression. Int J Mol Med. (2019) 44:2181-8. doi: 10.3892/ijmm.2019.4368

79. Wong $\mathrm{D}$, Yiu WH, Chan KW, Li Y, Li B, Lok S, et al. Activated renal tubular Wnt/beta-catenin signaling triggers renal inflammation during overload proteinuria. Kidney Int. (2018) 93:1367-83. doi: 10.1016/j.kint.2017. 12.017

80. Tse GH, Johnston CJC, Kluth D, Gray M, Gray D, Hughes J, et al. Intrarenal $\mathrm{B}$ cell cytokines promote transplant fibrosis and tubular atrophy. Am J Transplant. (2015) 15:3067-80. doi: 10.1111/ajt.13393

81. Nishida $A$, Inatomi $O$, Fujimoto $T$, Imaeda $H$, Tani $M$, Andoh $A$ Interleukin-36 $\alpha$ induces inflammatory mediators from human pancreatic myofibroblasts via a MyD88 dependent pathway. Pancreas. (2017) 46:53948. doi: 10.1097/MPA.0000000000000765

82. Zhao R, Dong R, Yang Y, Wang Y, Ma J, Wang J, et al. MicroRNA155 modulates bile duct inflammation by targeting the suppressor of 
cytokine signaling 1 in biliary atresia. Pediatr Res. (2017) 82:100716. doi: 10.1038/pr.2017.87

83. Chen K, Wei Y, Alter A, Sharp GC, Braley-Mullen H. Chemokine expression during development of fibrosis versus resolution in a murine model of granulomatous experimental autoimmune thyroiditis. J Leukocyte Biol. (2005) 78:716-24. doi: 10.1189/jlb.0205102

84. Zhu X, Sun Y, Mu X, Guo P, Gao F, Zhang J, et al. Phospholipase C $\varepsilon$ deficiency delays the early stage of cutaneous wound healing and attenuates scar formation in mice. Biochem Biophys Res Commun. (2017) 484:14451. doi: 10.1016/j.bbrc.2017.01.054
Conflict of Interest: The authors declare that the research was conducted in the absence of any commercial or financial relationships that could be construed as a potential conflict of interest.

Copyright $\odot 2021 \mathrm{Wu}$, Yin, Wang and Ying. This is an open-access article distributed under the terms of the Creative Commons Attribution License (CC BY). The use, distribution or reproduction in other forums is permitted, provided the original author(s) and the copyright owner(s) are credited and that the original publication in this journal is cited, in accordance with accepted academic practice. No use, distribution or reproduction is permitted which does not comply with these terms. 